\title{
Limited laser treatment for retinopathy of prematurity: A new hypothesis for pathogenesis of ROP based on ambiguity of vitreoretinal interface, immaturity of internal limiting membrane and mis-retinalization
}

\author{
Huseyin Yetik ${ }^{1,2 *}$ \\ ${ }^{1}$ Istanbul University, Cerrahpasa School Of Medicine, Fatih, Istanbul, Turkey \\ ${ }^{2}$ Professor Yetik Retina Clinic, Nurol Tower 1604, Istanbul, Turkey
}

\begin{abstract}
Purpose: To research the efficacy of limited laser treatment confined to the demarcation area between vascular and avascular retina thereby to determine the role of avascular retina, ischemic stimulus, neovascular response cascade in ROP.

Procedures: This is a prospective interventional case series study performed in a clinical practice setting. Total $68 \mathrm{ROP}$ patients $(\mathrm{N}=68,136$ eyes) with $29.338 \pm 1.441$ (26 to 32 ) weeks of gestational age and $1278.823 \pm 266.886$ (700 to 1800) grams of birth weight having type-1 pre-threshold disease all in zone-II needing treatment bilaterally were included. One eye of each patient randomly assigned as study eyes $(\mathrm{N}=68)$ and fellow eyes $(\mathrm{N}=68)$ as controls. For study eyes a limited laser treatment with dense laser spots completely covering the demarcation line (stage II /III) 360 degrees around 5-6 rows extending to both at vascular and avascular sides by leaving the most of peripheral avascular retina free of laser was performed and fellow control eyes got a standard pan-avascular-retinal laser treatment. Patients were evaluated with one to two week intervals until complete regression of the ROP occurred and/or for study eyes until complete vascularization of avascular retina achieved. All patients were followed up for $24.235 \pm 6.494$ (12 to 36) months. Regression of ROP as regression of plus and proliferations, maturation of retina and complications were evaluated. Chi Square and t-tests ( $<<0.05,95 \%$ confidence interval) were used for statistical analysis.

Results: Number of laser spots and time duration of laser session applied to the study and the control eyes were mean $415.088 \pm 74.092$ (290 to 600) spots in mean $6.015 \pm 1.263$ (4 to 9 ) minutes and $1930.808 \pm 322.547$ (1350 to 2700 ) spots in $16.455 \pm 1.980$ (14 to 25$)$ minutes, respectively and the difference in both number of laser spots and time durations were significantly different $(\mathrm{p}=0.000, \mathrm{t}$-test) between the groups. At the end of the first week $4 / 68(5.88 \%)$ study eyes $2 / 68(2.94 \%)$ control eyes needed additional laser treatment and the difference was not statistically significant ( $\mathrm{p}=0.404$, Chi Square test). During follow up period 3/68 (4.41\%) study eyes developed a secondary demarcation line but without a need for treatment regressed spontaneously. Total regression was achieved in all eyes and no recurrence was observed at the end of the follow up period.

Conclusions: Limited laser treatment covering demarcation line 360 degrees 5-6 rows with significantly less number of laser spots in significantly shorter duration of time can treat ROP disease as successful as pan-avascular ablation for zone-II, type-1 pre-threshold cases. Limited laser treatment can save more retinal tissue without ablation and significantly less duration of time of laser session applies significantly less systemic stress on premature infant. On the other hand it can be speculated that ROP may be a localized disease of eye confined to the vitreoretinal interface at least at its initial stages and it might not be a neovascular disease but intravitreally grown aberrant normal vasculature. Namely mis-retinalization hypothesis we constructed claims the main pathological origin of ROP to be the ambiguity of premature vitreoretinal interface with immature ILM that could not guide the retinal vasculogenesis to be confined to the retinal plane resulting diverged vasculogenesis falsely grown into the vitreous gel.
\end{abstract}

\section{Introduction}

Retinopathy of prematurity (ROP), formerly known as retrolental fibroplasia because of its end-stage appearance, is a developmental vascular proliferative disorder that occurs in the retina of preterm infants with incomplete retinal vascularization [1]. Next to cortical blindness, ROP is the most common cause of childhood blindness in the United States [2].

Although the pathogenesis of ROP is not well understood the mechanism is thought to involve two stages. An initial injury caused by factors such as hypotension, hypoxia, or hyperoxia, with free radical formation, injures newly developing blood vessels and disrupts normal angiogenesis. Following this disruption, vessels either resume normal growth or new vessels grow abnormally out from the retina into the vitreous. Increased permeability of these abnormal new vessels (neovascularization) can result in retinal edema and hemorrhage. Abnormal fibrovascular tissue may develop along with

Correspondence to: Huseyin Yetik, Ophthalmologist. Associate Professor of Ophthalmology, Ataköy 2-5-6. Mah. Beyaz Lale Sokak, Novus Residence No: 4/2 D-1 Blok Daire: 26, 34158, Bakirkoy, İstanbul, Turkey, Tel: 00905322820047; Fax: 00902125825053; E-mails: huseyinyetik@gmail.com, huseyinyetik@icloud.com

Key words: aberrant vascularization of vitreous, ambiguity of vitreoretinal interface, internal limiting membrane maturation, mis-retinalization, retinopathy of prematurity (ROP)

Received: September 21, 2016; Accepted: October 20, 2016; Published: October 24,2016 
Yetik H (2016) Limited laser treatment for retinopathy of prematurity: A new hypothesis for pathogenesis of ROP based on ambiguity of vitreoretinal interface, immaturity of internal limiting membrane and mis-retinalization

the neovascularization and later contract, producing traction on the retina. In some severe cases, this results in retinal distortion or retinal detachment [3-5].

Regulation of the expression of vascular endothelial growth factor (VEGF) and other cytokines like insulin-like growth factor-1 (IGF-1) may contribute to both normal retinal vessel growth and abnormal vascular disruption and subsequent neovascularization [5-12].

Therefore to block the ischemic neovascular stimulus i.e. to block or decrease the production of VEGF, standard treatment consists of complete ablation of the peripheral avascular retina, the source of ischemic neovascular stimulus, by cryotherapy [13] or laser [14] photocoagulation. Retinal ablative therapy reduces the incidence of adverse structural and functional outcomes $[13,14]$.

However if it is an ischemic stimulus-neovascular response disease like diabetic retinopathy or retinal vein occlusions, a neovascular response like neovascularisation of elsewhere (NVE), neovascularisation of disc (NVD), neovascularisation of iris (NVI) and neovascular glaucoma (NVG) etc. might be expected as well. But even in the worst stages of the disease it is not the case.

Furthermore in our more than 10 years of clinical experience including approximately 50 premature infants examinations per week, despite almost total avascular peripheral retinal ablation, if there remained a very tiny non-lasered area closely in front of the demarcation line with neo-vascular proliferations on, treatment was failing particularly at that retinal segment. In addition, best responses were achieved at the areas where the laser spots had covered the demarcation line (stage II or even III) totally (Figure 1).

Those clinical observations have aroused the question if a generalized or localized response of retina was dominant in the pathogenesis of the disease and pushed us in suspicion about the exact role of ischemic stimulus-neovascular response cascade in the pathogenesis of ROP.

Therefore to explain those clinical observations and to clarify the exact role of avascular retina in conjunction with ischemic stimulus neovascular response cascade in the pathogenesis of ROP we designed this study.

\section{Materials and methods}

All parents signed an informed consent that is prepared according to the principles outlined in the Declaration of Helsinki and approved by the ethical committee of Cerrahpasa Medical Faculty. During the period of January 2009 to January 2012, with a design of prospective

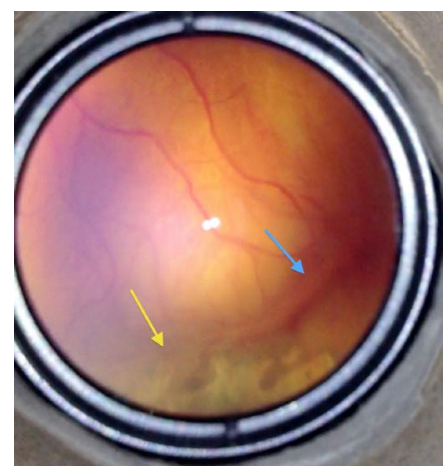

Figure 1. Typical picture of a remaining stage 3 disease after laser treatment at a sector with a very tiny non-lasered area in front of it (blue arrow) but totally regressed at the sector where laser burns covered the demarcation line totally (yellow arrow). interventional case series study performed under a clinical practice setting, total 68 ROP patients ( $\mathrm{N}=68,136$ eyes) with $29.338 \pm 1.441(26$ to 32 ) weeks of gestational age and $1278.823 \pm 266.886$ (700 to 1800) grams of birth weight having type-1 pre-threshold disease all being in zone-II and needing treatment bilaterally were included.

Without any masking procedure one eye of each patient randomly assigned as study eye $(\mathrm{N}=68)$ and fellow eye $(\mathrm{N}=68)$ as controls.

For study eyes a limited laser treatment with dense laser spots completely covering the demarcation line (stage II /III) 360 degrees around 5-6 rows extending to both vascular and avascular sides by leaving the most of peripheral avascular retina free of laser was performed (Figure 2 and 3). Fellow control eyes got a standard panavascular-retinal laser treatment.
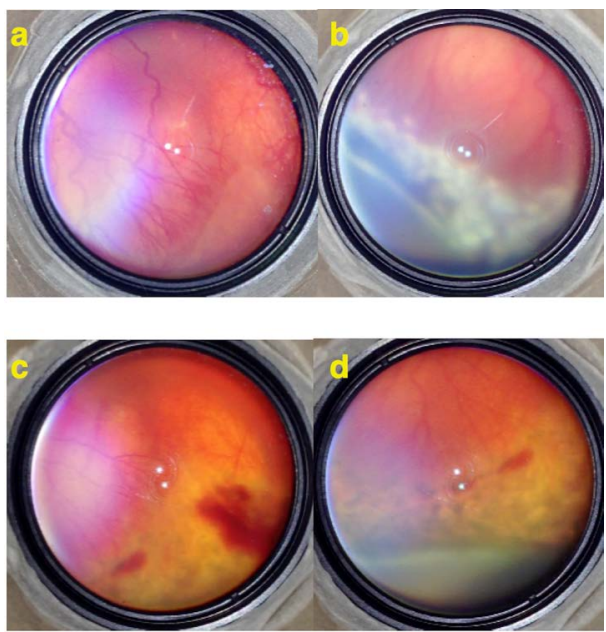

Figure 2. a) Stage 3 ROP with plus disease. Note the (neo)vascularizations extend as normal continuation of retinal vessels. b) Limited laser treatment of stage 3 area by white (fresh) laser spots extending over both vascular and avascular sides of demarcation but leaving most of peripheral avascular retina free of laser. c) 1 week after the limited laser treatment. A limited intravitreal hemorrhage occurred and proliferations and plus disease are regressed. d) 3 weeks after the laser treatment. Laser spots became more pigmented and the disease is totally regressed.

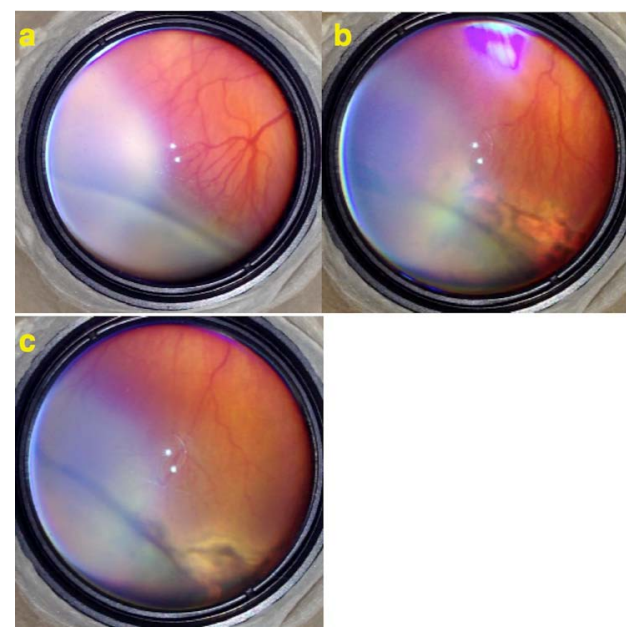

Figure 3. a) Stage 3 ROP with plus disease. Neo-vascularizations extend as norma continuation of branching of retinal vessels. Note that instead of having normal dichotomic branching retinal vessels split into multiple branches which is very typical for ROP b) 2 weeks after the limited laser treatment plus disease and proliferations are regressed. c) 1 month after the laser. Laser scares are enlarged and became more pigmented and the disease is is totally regressed. 
Yetik H (2016) Limited laser treatment for retinopathy of prematurity: A new hypothesis for pathogenesis of ROP based on ambiguity of vitreoretinal interface, immaturity of internal limiting membrane and mis-retinalization

Number of laser spots and time duration of laser session applied to the study and control eyes were compared and regression of ROP as regression of plus and proliferations, maturation of retina and complications were evaluated as main outcome measures.

Patients were evaluated with one or two weeks' intervals until complete regression of the ROP occurred and/or for study eyes until complete vascularization of avascular retina achieved and thereafter with 3 or 6 monthly interval throughout the follow up period. All patients were followed up for $24.235 \pm 6.494$ (12 to 36) months. Chi Square test $(\mathrm{p}<0.05,95 \%$ confidence interval) was used for statistical analysis.

\section{Results}

Number of laser spots and time duration of laser session applied to the study and the control eyes were mean $415.088 \pm 74.092$ (290 to 600) spots in mean $6.015 \pm 1.263$ (4 to 9) minutes and $1930.808 \pm 322.547$ (1350 to 2700 ) spots and $16.455 \pm 1.980$ (14 to 25 ) minutes respectively and the difference in both number of laser spots and time durations were significantly different ( $\mathrm{p}=0.000$, $\mathrm{t}$-test) between the groups.

At the end of the first week 4/68 (5.88\%) study eyes and 2/68 (2.94\%) control eyes needed additional laser treatment because of incomplete regression of ROP and the difference was not statistically significant ( $\mathrm{p}=0.404$, Chi Square test $\mathrm{p}<0.05,95 \%$ confidence interval).

During follow up period 3/68 (4.41\%) study eyes developed a secondary demarcation line and without a need for laser treatment it regressed spontaneously and retinal vascularization completed without any further complication (Figures 2 and 3).

Total regression of the disease was achieved in all eyes and no recurrence was observed at the end of the follow up period. After limited laser treatment of demarcation area in study eyes normal retinal vascularization continued to progress and crossed the laser treated areas to vascularize the peripheral retina completely.

\section{Discussion}

Results of the study reveals that at least for Zon-II type-1 prethresold cases limited laser treatment confined to the demarcation area extending to both vascular and avascular sides can regress the disease as successful as panavascular laser ablation. In comparison to pan-avascular laser treatment, limited laser treatment was performed by a significantly less number of laser spots, mean 415.088 versus 1930.808 , in a significantly shorter duration of time, 6.015 versus 16.455 minutes. Therefore, limited laser treatment applies significantly less stress on premature infant as well.

Confirming our years of observation ablating the area that stands as a source or base for intravitreal vascular proliferations could terminate the progression of the disease i.e., stage-3 and plus disease (Figure 2 and 3) (See Supplemental Video 1).

Depending upon those results we speculate that localized and limited laser treatment of 5-6 rows covering the demarcation line can control the pathological vascular growth into the vitreous body and regress the ROP disease.

More interestingly, normal vascular maturation of previous avascular retina is possible by the vessels crossing over the limited laser area (Figure 2-3). On the other hand, despite successful regression of the ROP after the laser ablation of the demarcation line there is still a possibility to develop a second demarcation line until the vasculature

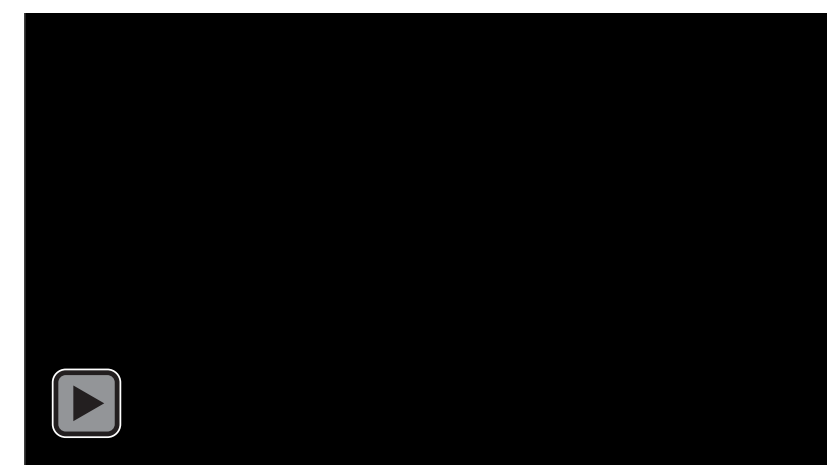

Supplemental Video 1

Limited Laser of ROP: This video shows pre- and post-laser video images of some cases from the study. Regression of the disease as regression of plus and proliferations is obvious.

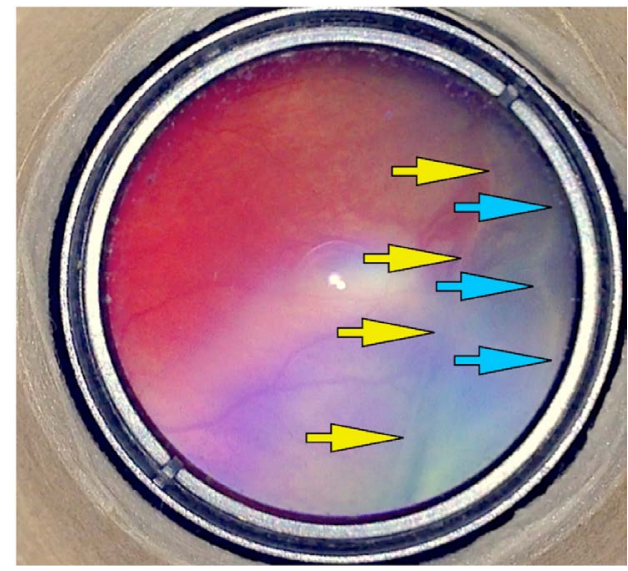

Figure 4. A typical picture of a ROP supporting the main claim of the Vitreoretinal Interface Immaturity and Mis-retinalization Hypothesis. There can be seen 2 demarcation lines pointed by yellow and blue arrows. Yellow arrows point the previous demarcation line where the disease progressed to stage 3 but when ILM and vitreoretinal interface maturation occur aberrant vessels stop to grow into the vitreous and continue to grow back into the retinal plane forwardly. Normal retinal vessels crossed the demarcation line pointed by yellow arrows and previous stage 3 disease converted into vitreous opacifications. But after a while retinal vessels come to another border of vitreoretinal interface ambiguity and construct another demarcation line. Those progression and regression forms are typical and unique to ROP not seen in another ischemic VEGF related neovascular vitreoretinal disease. ROP is a unique disease and it resembles only to itself.

reach to the ora serrata (Figure 4).

Since a laser ablation limited to the level of demarcation line extending to both vascular and avascular sides can regress the disease then this supports our 2 previous observations:

1) Avascular retina - ischemic stimulus - neovascular response cascade might not have a significant role -if there is any- in the pathogenesis of ROP

2) ROP might not be a disease having a generalized retinal response to a generalized retinal stimulus. In other words, ROP might be a more localized retinal pathology confined to the demarcation line at least in its initial stages.

As a combination of all those observations and some known other issues written in the literature primary author (Dr. H. Yetik) constructed a new hypothesis that he named as Vitreoretinal Interface Ambiguity \& Mis-retinalization Hypothesis.

It is a fact that the pathogenesis of ROP is not well understood. None of the present hypothesis or theory of ROP is able to explain all the clinical features of ROP [3-12]. If ROP is a kind of ischemic- 
Yetik H (2016) Limited laser treatment for retinopathy of prematurity: A new hypothesis for pathogenesis of ROP based on ambiguity of vitreoretinal interface, immaturity of internal limiting membrane and mis-retinalization

neovascular disease of retina why there is no NVE, NVD or NVG even in its most advanced stages? Furthermore why there are some unique clinical stages (stage 1 to 5 ) as well as unique spontaneous regression features like vessels crossing demarcation line, regressed stage-3 areas separated from retinal surface those not seen in other ischemic proliferative retinal diseases $[15,16]$.

In our opinion all the present hypothesis or theories are ignoring the main issue of ROP which is prematurity itself. For instance as we mentioned above regulation of the expression of vascular endothelial growth factor (VEGF) and other cytokines may contribute to both normal retinal vessel growth and abnormal vascular disruption and subsequent neovascularization but we do not know if the responses of mature and immature retinal tissues are the same to those cytokines.

In addition none of other hypothesis or theories of ROP dealt with tissue maturation process including ambiguity and differentiation. Whereas differentiation is a highly controlled process essential for embryonic and adult development. Moreover, disruption of proper differentiation is often associated with human diseases, including cancer $[17,18]$. Mostly known one is ambiguous genitalia in which the fate of somatic precursor of gonads the genital ridge governed by the differentiation of somatic cells into Sertoli cells in the testes or granulosa cells in the ovaries further determines the sex of an individual and their germ cells disrupts [19].

However this new hypothesis mainly stands on ambiguity and differentiation of tissues and tissue planes and suggests 2 main pathophysiological mechanisms (Figure 5):

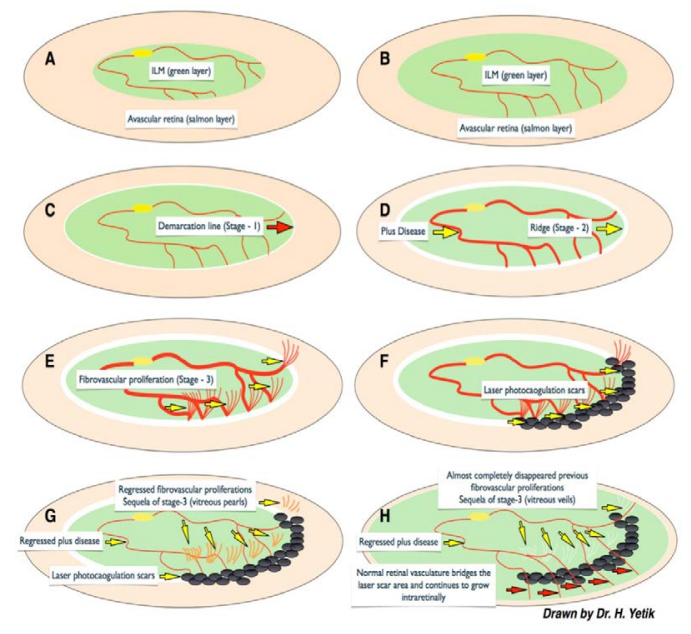

Figure 5. A,B) According to this new hypothesis ILM (green layer) maturation and ambiguity of vitreoretinal interface drives a primary role in the pathogenesis of ROP. Maturation and peripheral progression of ILM both delineates the vitreoretinal interface and serves as a guide to retinal vasculogenesis to be confined into the retinal plane. C)If ILM or vitreoretinal interface maturation disrupts then the vitreoretinal interface stays ambiguous and glial tissue of ILM material starts to collect in front of the ambiguous vitreoretinal plane that is seen as a white demarcation line combining the tips of the vessels known as Stage - 1. D) If the disease continues to progress then ILM material collects more and gets an appearance of ridge known as Stage 2. Microvasculature formation between artery and vein behind the ridge causes a short-circuit between arterial and venous system which results into the "plus disease". E) If the ambiguity of vitreoretinal interface persists and ILM can not guide the vascular maturation then progression of retinal vasculature lose the intraretinal plane and diverge to grow into the gel vitreous which is seen as fibrovascular proliferation, Stage-3. F) If laser photocoagulation enough to cease the vascular growth into the viteous gel is applied onto the demarcation area covering both vascular and avascular sides then retinal vasculature can not progress into the vitreous gel that gives time to ILM and vitreoretinal interface to mature more. G) If the disease resolves either spontaneously or after laser treatment, plus disease and fibrovascular proliferations regress and sequel of Stage -3 fade and separate from retinal surface thoseremains as free vitreous opacities aligned like the prayer beads which we named as "vitreous pearls". H) Weeks after a successful limited laser treatment, aberrant fibrovascular proliferation completely resolves and normal vasculature continues to grow into the retinal plane by bridging the laser scar area.

\section{Ambiguity of Vitreoretinal Interface and ILM Immaturity}

\section{Vascular aberration and mis-retinalization of vitreous gel}

\section{Ambiguity of vitreoretinal interface and ILM immaturity}

As a general rule of maturation, more maturity should mean more differentiation of tissues; in other words, more immaturity should mean less differentiation or more ambiguity of tissues and tissue planes. Therefore, more mature the eye means more differentiated or delineated the ocular tissues and tissue planes.

For a living body demarcation of ocular tissue planes is a lifetime continuing process especially at the vitreoretinal interface. That is why it is too hard to separate posterior hyaloid during vitrectomy surgery of a child [20] but $75 \%$ of population over 65 years of age experience spontaneous posterior vitreous detachment (PVD) [21]. Then it is logical and scientific to say that vitreoretinal interface differentiation is a continuously ongoing process and it is less differentiated or more ambiguous in childhood and maybe almost undifferentiated in a premature infant.

Since the main tissue defining and determining retinal limits to the posterior vitreous surface i.e. the vitreoretinal interface is internal limiting membrane (ILM) then vitreoretinal interface maturation or differentiation should depend upon the maturation of this main demarcating tissue. Therefore if we mention about the maturation of vitreoretinal interface we should mention about the maturation of this main demarcating tissue plan, which is "internal limiting membrane" as well.

During an ophthalmoscopy, healthy and mature retina has a bright light reflex under ophthalmoscopic light. This bright glistening emerges from internal limiting membrane (ILM). We can confirm this from nowadays' popular vitreoretinal technique of "ILM peeling". During ILM peeling with or without any staining technique, under microscopic high magnification and endoillumination it is very easy to differentiate ILM as a thin glistening elastic lacquer-like tissue covering the retinal surface. If it is pinched with forceps it wrinkles easily like a transparent cellophane membrane and it can be torn like anterior capsule of crystalline lens [22-24].

In a premature infant with an immature retina it is not possible to see a healthy bright retinal light reflex from retinal surface under indirect ophthalmoscopic illumination. By the time as the retina and ILM matures bright light reflex from retinal surface becomes visible at the mature vascular retinal area posterior to the demarcation line but avascular retina anterior to the demarcation stays matt (Figure 6).

Therefore retinal maturation process may be imagined like a production of a knitted tissue together with vascular and neural elements and the surface of final knitted tissue is covered with internal limiting membrane while maturation progresses.

\section{Vascular aberration (aberrant vasculogenesis) and mis- retinalization of vitreous gel}

Because of the immaturity or in other words "ambiguity" of vitreoretinal interface the progressing normal retinal vasculature lose its true intraretinal plane and thereafter continue to grow into the vitreous body pathologically or aberrantly.

Fibrovascular proliferation in stage 3 ROP is a kind aberrant vascular proliferation growing into the gel vitreous instead of being a neovascular proliferation. In other words normal retinal vascularization 


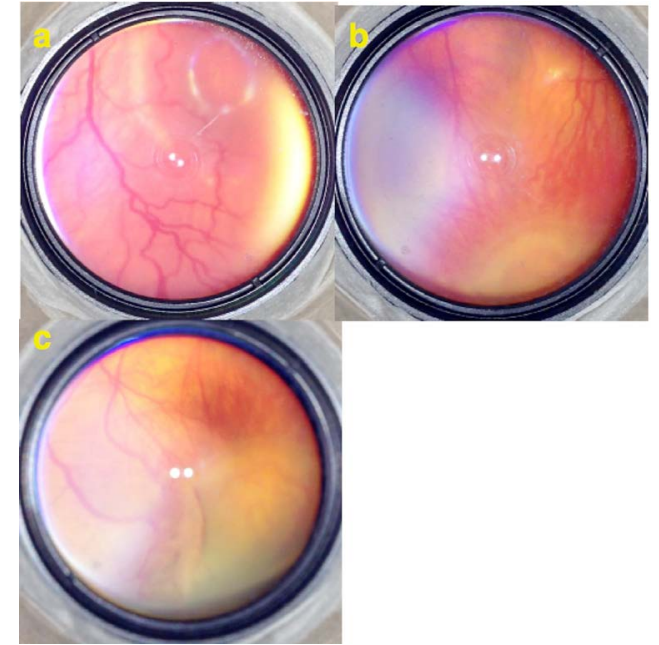

Figure 6. a) A brilliant reflection of ophthalmoscopic light from the retinal surface especially around the foveal umbo can be seen easily. This glistening reflection emerges from mature ILM. b-c) A bare light reflection from the retinal surface posterior to the aggressive stage 3 disease where the mature ILM is present can be seen but the surface of the stage 3 and avascular area where the ILM is absent or immature seems matt.

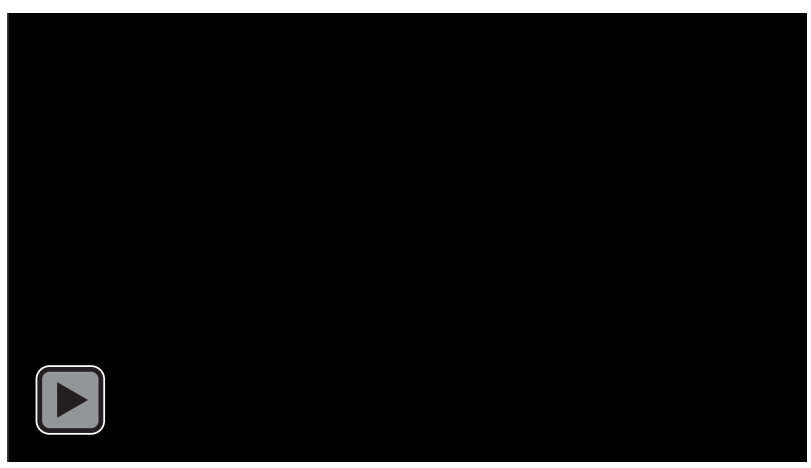

Supplemental video 2

Very Serious ROP Stage 3: This video shows a very serious form of ROP having almost 360 degrees of stage $3+$ disease and scattered vitreous hemorrhages.

loses its true intraretinal pathway and thereafter continues to grow into the gel vitreous. Therefore it is a misdirection of normal retinal vascular maturation and that's why in its extreme form 360 degrees of vascular proliferation called "purse string membrane" occurs (See Supplemental video 2) (Figure 5). This type of fibrovascular proliferation is not typical for any other ischemic neovascular proliferative disease of retina.

One of the other main clue supporting this hypothesis is those intravitreally growing pathological vasculature seems like a normal continuation of retinal vasculature extending into the gel vitreous. In fact, vasculature continues to grow into the vitreous gel as it is retinal plane mistakenly and usually inexperienced eyes interpret those intravitreal aberrant vessels as the retina itself and mis-diagnose a traction retinal detachment. Because of its appearance as being a normal continuation of retinal vasculature intravitreal aberrant vascular network seems uniformly as retinal vasculature that's why the hypothesis was named as mis-retinalization (or pseudo-retinalization) of vitreous gel. This is particularly obvious during vitrectomy surgery for ROP during which intravitreal vascular network of ROP constructed as a continuation of main vascular arcades of retina can be seen obviously. Interestingly if you cut those mis-retinal vessels extending into the vitreous gel by vitreous cutter they do not bleed aggressively. Even during the vitrectomy surgery sometimes it may be very difficult to differentiate those mis-retinal vessels and mis-retinal plane from the real retina. But as the surgical time passes vitreous gel becomes more hydrated and it becomes easier to differentiate the real retina from misretina (Figure 7) (See Supplemental video 3).

Interestingly this is very consistent with the ultrastructural features of the ROP membranes as well. Because in ultrastructural studies it was shown that retinal glial cells (presumably Müller cells) were seen fragmenting the internal limiting lamina and migrating into the overlying vitreous by projecting long microvilli into the vitreous cavity and vitreal neovascularisation was found to break through the internal limiting membrane $[25,26]$. Therefore there is really a misretinalization of vitreous gel since as being the principal glial cells of retina, Muller cells are both the elements of the retina as well as the internal limiting membrane. Normally Muller cells would form the ILM and by the way would define the internal retinal boundary and confine the retinal vasculature to the retinal plane. But ambiguity of vitreoretinal plane or failure to define and determine the vitreoretinal interface truly exerts the retinal vasculature to grow into the vitreous gel aberrantly.

At any stage of the disease if internal limiting membrane can

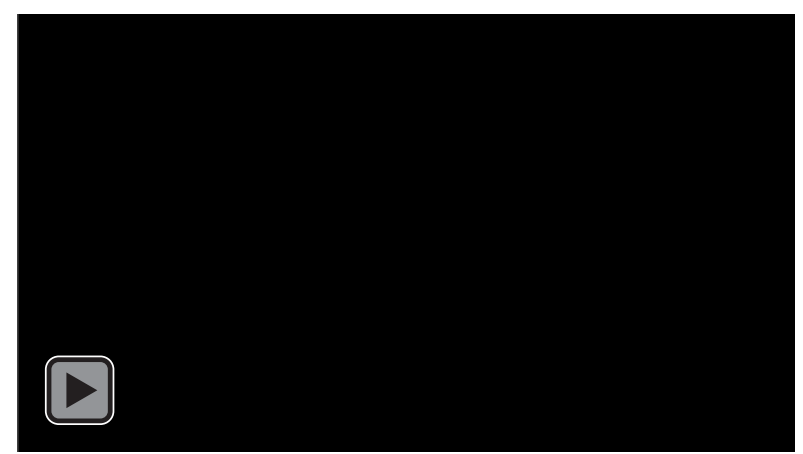

Supplemental video 3

Aberrant Vasculogenesis During Vitrectomy: This video shows apparently the vascular network of retinal vessels of aberrantly grown into the vitreous gel and having the appearance of mis-retinalization and pseudoretinalization which may cause a misdiagnose of retinal detachment.
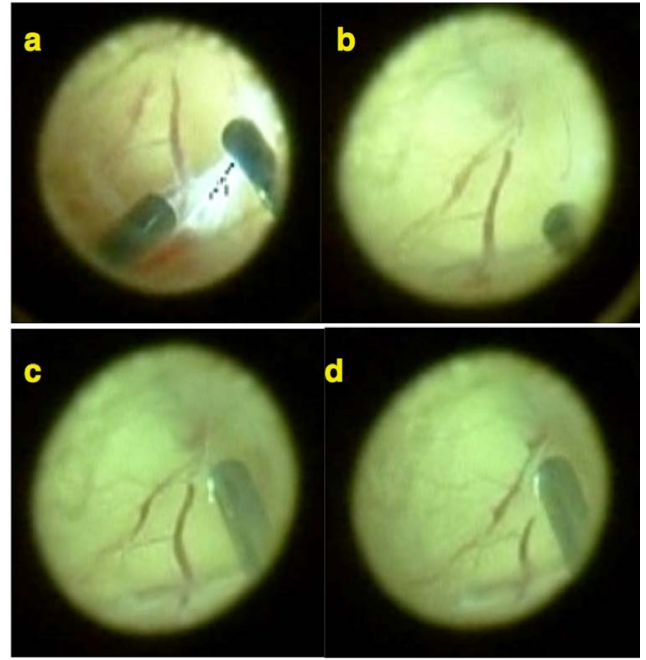

Figure 7. a-b-c) Typical picture of mis--retinalization of vitreous taken during a vitrectomy operation (Operator Dr HuseyinYetik). Retinal vessels diverged and branched into the vitreous gel can be seen.c-d)Those aberrant vessels do not bleed when cut by vitreous cutter. 
Yetik H (2016) Limited laser treatment for retinopathy of prematurity: A new hypothesis for pathogenesis of ROP based on ambiguity of vitreoretinal interface, immaturity of internal limiting membrane and mis-retinalization

mature and cover the vascularized retinal surface to define the true vitreoretinal interface then it can cleave the true vitreoretinal plane and cut off the false plane that is crossed by the aberrant vasculature grown into the vitreous gel. Thereafter the retinal vasculature becomes aware of the pathological vitreal plane and grows back into the normal retinal plane. Previous fibrovascular tissue (stage 3) spontaneously regresses by transforming into ghost vessel and vitreous opacities and then disappears (Figure 4,5). This process is visible in fundus examination as normal retinal vasculature crosses the demarcation line, previous stage 3 disease separates form retinal plane and transforms into ghost vessels as well as vitreous opacities aligned linearly like prayer beads we named this picture as "vitreous pearls" (Figure 5). Those signs almost always indicate regression in the disease and are unique for ROP. None of the other ischemic neovascular retinal disease has those regression characteristics. For example in diabetic retinopathy, we can see intraretinal collector canals as intraretinal microvascular abnormalities (IRMAs) but instead of being a good sign it is a sign of a more complicating and severe disease.

Anatomically larger retinal vasculature is exactly located on the retinal surface and covered by ILM. We know this anatomical reality from traction vitreoretinal diseases i.e. proliferative diabetic retinopathy which may cause separate detachment of retinal vessels from retinal surface as well as from sheathotomy surgery that aims to cut common arterial-venular sheath of the retinal vessels at the arteriovenous crossing areas in which surgical instruments are inserted between retinal surface and retinal vessels. Therefore, as a process of maturation retinal vasculogenesis should occur on the retinal surface but micro-capillaries should penetrate the retinal tissue. This superficial anatomical localization of the retinal vasculature also supports the logic behind this new Vitreoretinal Interface Immaturity \& Mis-retinalization Hypothesis of the study.

Furthermore this new hypothesis is able to explain all ROP stages besides unique regression characteristics of the disease (Figure 5). Those stages are unique for ROP in comparison to other ischemia neovascularisation cascade type retinal diseases. In fact presence of those unique stages in comparison to other neovascular vitreoretinal diseases solely ascertains the need for a different pathogenesis of ROP.

According to this new hypothesis at initial phases of the disease maturing ILM material - probably glial elements of Muller cells- those are unable to cleave the vitreoretinal interface start to collect at the border of ambiguous vitreoretinal plane linearly. This linear white collection represents the "demarcation line" which is termed as Stage I. By the time this material continue to collect more as well as the vasculogenesis unable to go further peripherally deviates and a shunting occurs between the arterial and venous sides and those process are seen clinically as "ridge-like" configuration of previous demarcation which is termed as stage 2 and engorgement and tortuosity of the retinal vasculature which is known as "plus disease" respectively. If this pathological process continue then deviated vasculogenesis continues intravitreally which is termed as Stage 3 and might face a more tissue resistance in the vitreous in comparison to retinal tissue plane which may be another reason underlying the plus disease. Finally, if the aberrant vasculogenesis into the vitreous gel continues after a critical point it becomes impossible to regress and progresses to stage IV and stage $\mathrm{V}$ respectively (Figure 5).

On the other hand, in the active stages of the disease, pupillary rigidity and rubeosis iridis are seen but they are not like classical neovascularisation of iris as well. Because in its natural course, if there is no operation performed, even in the final stages of the disease it is almost impossible to see a neovascular glaucoma complication in a ROP case. Also there is usually no synechia posterior if there is no laser burn at the pupillary margin as well as it is still possible to dilate those rubeotic pupils and there is no inflammatory membrane on the iris surface that cause ectropion of iris. Furthermore, pupillary rubeosis disappers in seconds after opening sclerotomies during ROP vitrectomy (Figure 8) (Supplemental Video 4). Therefore, instead of being a neovascularisation, rubeosis iridis in ROP is more possible to be a recanalization tunica vasculosa lentis.

Finally this new hypothesis is able to explain the successful results of anti-VEGF treatment (intravitreal bevacizumab - IVB) besides. If aberrant vasculogenesis into the vitreous gel is suppressed and/or regressed by anti-VEGF agents it simultaneously gives time for ILM maturation in other words maturation of vitreoretinal interface as well. Therefore, any intervention delaying the retinal vasculogenesis until the vitreoretinal interface maturation completes it can have a potential to treat ROP.

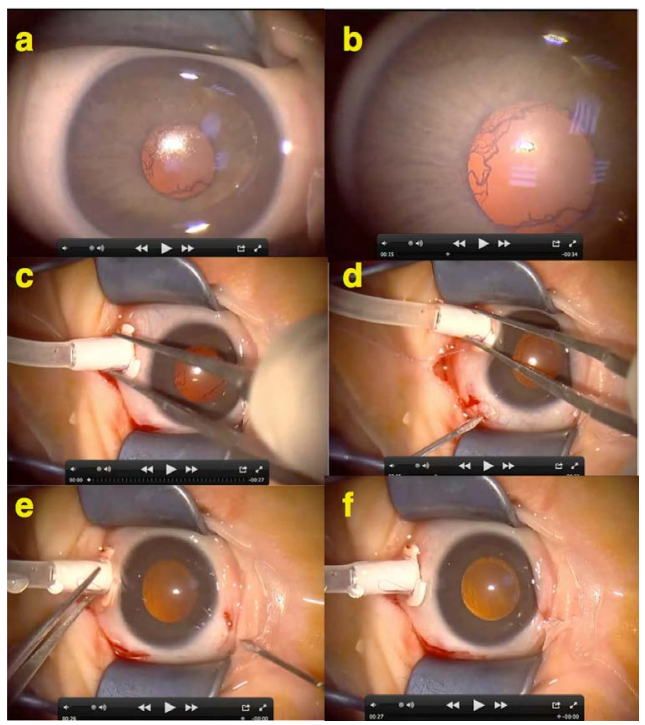

Figure 8. This figure shows the rubeosis iridis (RI) seen in ROP is not like a neovascula response of anterior segment as in other ischemia - VEGF related neovascular diseases of retina.a-b) At the beginning of the operation RI is obvious but typically vessels at the pupillary borders seems as normal continuation of radial iris vessels. c-d-e-f) Seconds after the opening sclerotomies RI disappears suddenly. Therefore RI seen in ROP is more likely to be a "recanalization of tunica vasculosalentis".

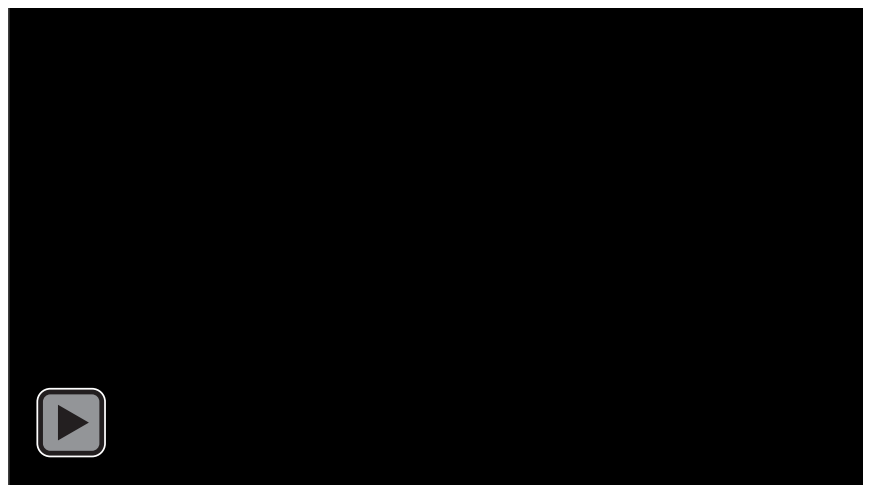

Supplemental Video 4

Rubeosis Iridis of ROP: This videos shows the sudden disappearance of rubeosis iridis at the beginning of a ROP vitrectomy seconds after the openings of sclerotomies and supporting the idea of rubeosis iridis in ROP is more likely to be a recanalization of tunica vasculosalentis instead of being a true neovascularization of iris. 
Yetik H (2016) Limited laser treatment for retinopathy of prematurity: A new hypothesis for pathogenesis of ROP based on ambiguity of vitreoretinal interface, immaturity of internal limiting membrane and mis-retinalization

\section{Conclusions}

In conclusion results of this study show that ROP is a more localized disease of retina and confined to vitreoretinal interface at least at its initial stages. ROP might not be an ischemic -neovascular disease of retina; instead of being neovascularisation normal but aberrantly grown vasculature may be the main vascular pathology seen in the disease.

Now then as shown in the above explanations our new vitreoretinal interface immaturity \& misretinalization (psudoretinalization) hypothesis (of Dr. H.Y.) is able to explain and combine scientifically almost all of the literature data and clinical presenting and regressing signs of ROP despite some of them are unexplained by presently known hypothesis or theories of ROP yet. ROP is exactly a unique disease and it resembles only to itself. We believe that the logic and mechanisms behind this new Vitreoretinal Interface Ambiguity \& Mis-retinalization Hypothesis of ROP are worth to be considered, evaluated and discussed further by the scientific community of ophthalmology worldwide.

\section{Financial support}

Governmental Scientific and Technological Research Council of Turkey (TUBITAK) supported the development and patenting process of Dr. Huseyin Yetik's Medical Imaging Device used in this study and TUBITAK had no role in the design or conduct of this research.

\section{Conflict of interest}

There is no conflicting relationship exists for any author.

\section{Previously presented in}

1. Limited laser treatment covering the demarcation line in retinopathy of prematurity - A new hypothesis in the pathogenesis of ROP. Euretina Conference: Paris 2010 Session: Vascular Diseases And Diabetic Retinopathy. Presenting Author: Huseyin Yetik.

2. Retinopathy of prematurity (ROP): What we know and what we should know about? Huseyin Yetik (Istanbul/TR). World Ophthalmology Congress 2010 ICC, Berlin, Germany XXXII International Congress of Ophthalmology 108th DOG Congress 5-9 June2010- Final Program Abstract Book- p.109).

\section{References}

1. Terry T (1942) Extreme prematurity and fibroplastic overgrowth of persistent vascular sheath behind each crystalline lens: I. Preliminary report. Am J Ophthalmol 25: 203.

2. Hamilton BE, Hoyert DL, Martin JA, Strobino DM, Guyer B (2013) Annual summary of vital statistics: 2010-2011. Pediatrics 131: 548-558.[Crossref]

3. Kretzer FL, Hittner HM (1988) Retinopathy of prematurity: clinical implications of retinal development. Arch Dis Child 63: 1151-1167.[Crossref]

4. Ashton N, Ward B, Serpell G (1954) Effect of oxygen on developing retinal vessels with particular reference to the problem of retrolental fibroplasia. Br J Ophthalmol 38: 397-432.[Crossref]

5. Young TL, Anthony DC, Pierce E, Foley E, Smith LE (1997) Histopathology and vascular endothelial growth factor in untreated and diode laser-treated retinopathy of prematurity. JAAPOS 1: 105-110.[Crossref]

6. Pierce EA, Foley ED, Smith LE (1996) Regulation of vascular endothelial growth factor by oxygen in a model of retinopathy of prematurity. Arch Ophthalmol 114: 12191228.[Crossref]

7. Stone J, Chan-Ling T, Pe'er J, Itin A, Gnessin H, et al. (1996) Roles of vascular endothelial growth factor and astrocyte degeneration in the genesis of retinopathy of prematurity. Invest Ophthalmol Vis Sci 37: 290-299.[Crossref]
8. Aiello LP, Northrup JM, Keyt BA, Takagi H, Iwamoto MA (1995) Hypoxic regulation of vascular endothelial growth factor in retinal cells. Arch Ophthalmol 113: 1538-1544. [Crossref]

9. Hellström A, Carlsson B, Niklasson A, Segnestam K, Boguszewski M, et al. (2002) IGF-I is critical for normal vascularization of the human retina. $J$ Clin Endocrinol Metab 87: 3413-3416.[Crossref]

10. Smith LE (2005) IGF-1 and retinopathy of prematurity in the preterm infant. Biol Neonate 88: 237-244.[Crossref]

11. Hellstrom A, Perruzzi C, Ju M, Engstrom E, Hard AL, et al. (2001) Low IGF-I suppresses VEGF-survival signaling in retinal endothelial cells: direct correlation with clinical retinopathy of prematurity. Proc Natl Acad Sci U S A 98: 5804-5808.[Crossref]

12. Hellström A, Engström E, Hård AL, Albertsson-Wikland K, Carlsson B, et al. (2003) Postnatal serum insulin-like growth factor I deficiency is associated with retinopathy of prematurity and other complications of premature birth. Pediatrics 112: 1016-1020. [Crossref]

13. [No authors listed] (1988) Multicenter trial of cryotherapy for retinopathy of prematurity. Preliminary results. Cryotherapy for Retinopathy of Prematurity Cooperative Group. Arch Ophthalmol 106: 471-479.[Crossref]

14. Early Treatment For Retinopathy Of Prematurity Cooperative Group (2003) Revised indications for the treatment of retinopathy of prematurity: results of the early treatment for retinopathy of prematurity randomized trial. Arch Ophthalmol 121: 1684-1694. [Crossref]

15. International Committee for the Classification of Retinopathy of Prematurity (2005) The International Classification of Retinopathy of Prematurity revisited. Arch Ophthalmol 123: 991-999.[Crossref]

16. Morizane H (1976) [Initial sign and clinical course of the most severe form of acute proliferative retrolental fibroplasia (type II) (author's transl)]. Nippon Ganka Gakkai Zasshi 80: 54-61.[Crossref]

17. Aylon Y, Sarver A1, Tovy A1, Ainbinder E2, Oren M1 (2014) Lats2 is critical for the pluripotency and proper differentiation of stem cells. Cell Death Differ 21: 624-633. [Crossref]

18. Regalo G, Leutz A (2013) Hacking cell differentiation: transcriptional rerouting in reprogramming, lineage infidelity and metaplasia. EMBO Mol Med 5: 1154-1164. [Crossref]

19. Tanaka SS, Nishinakamura R (2014) Regulation of male sex determination: genital ridge formation and Sry activation in mice. Cell Mol Life Sci 71: 4781-4802.[Crossref]

20. Yokoyama T, Kato T, Minamoto A, Sugihara A, Imada M, et al. (2004) Characteristics and surgical outcomes of paediatric retinal detachment. Eye (Lond) 18: 889-892. [Crossref]

21. Byer NE (1994) Natural history of posterior vitreous detachment with early management as the premier line of defense against retinal detachment. Ophthalmology 101: 1503-1513. [Crossref]

22. Sakimoto S, Ikuno Y, Fujimoto S, Sakaguchi H, Nishida K (2014) Characteristics of the Retinal Surface After Internal Limiting Membrane Peeling in Highly Myopic Eyes. $\mathrm{Am}$ J Ophthalmol 158: 762-768. [Crossref]

23. Walia HS, Shah GK, Hariprasad SM (2014) ILM peeling a vital intervention for many vitreoretinal disorders. Ophthalmic Surg Lasers Imaging Retina 45: 92-96.[Crossref]

24. Diaz RI, Randolph JC, Sigler EJ, Calzada JI (2014) Intraoperative grasp site correlation with morphologic changes in retinal nerve fiber layer after internal limiting membrane peeling. Ophthalmic Surg Lasers Imaging Retina 45: 45-49. [Crossref]

25. de Juan E, Gritz DC, Machemer R (1987) Ultrastructural characteristics of proliferative tissue in retinopathy of prematurity. Am J Ophthalmol 104: 149-156.[Crossref]

26. Yoneya S1, Tso MO (1991) Retinal and vitreal neovascularization in retinopathy of prematurity. A scanning electron microscopic study in the kitten. Arch Ophthalmol 109: 1744-1751.[Crossref]

Copyright: (C2016 Yetik H. This is an open-access article distributed under the terms of the Creative Commons Attribution License, which permits unrestricted use, distribution, and reproduction in any medium, provided the original author and source are credited. 